\title{
Alfabetización académica en la universidad: recursos literarios adicionales para aprender a investigar
}

\author{
María Isabel POZZO (D) \\ Universidad Nacional de Rosario y Universidad Tecnológica Nacional
}

\section{○ \\ OPEN ACCESS \\ EDITADO POR \\ - Eulália Leurquin \\ (UFC) \\ - Rita de Cássia Souto Maior \\ (UFAL) \\ - Matilde Alves Gonçalves (UNL) \\ AVALIADO POR \\ - Gonzalo Abio (UFAL) \\ - Kristianny B. B. de Azambuja (UFAL) \\ FECHAS \\ - Recebido: 14/09/2021 \\ - Aceito: 28/10/2021 \\ - Publicado: 23/12/2021 \\ COMO CITAR \\ Pozzo, M.I. (2021). \\ Alfabetización académica en la universidad: recursos literarios adicionales para aprender a investigar. Revista da Abralin, v. 20, n. 3, p. 1094-1103, 2021.}

\section{RESUMEN}

La universidad se caracteriza por la circulación y producción de ciertos géneros escritos (monografías, informes, proyectos) como uno de los modos privilegiados de evaluación de los aprendizajes. Aun tratándose de estudiantes que ya han superado los distintos niveles de la educación obligatoria, sus producciones escritas dejan ver deficiencias en cuanto a aspectos básicos de normativa de la lengua en general y del lenguaje académico en particular. Este diagnóstico afecta incluso a cursantes de carreras sociales y humanísticas en las que el lenguaje verbal es el medio habitual de estudio. Esta situación se magnifica al aprender a investigar, que se vale de la escritura para la socialización de los resultados. La formación en investigación discurre habitualmente entre la lectura de artículos de la especialidad, manuales de metodología y explicaciones magistrales. Sean las fuentes orales o escritas, se trata de textos principalmente instructivos de marcado corte cientificista, tendientes a sumergir a los estudiantes en las objetividad y neutralidad del discurso científico. Sin pretender ignorar esta bibliografía tradicional, el presente artículo propone abonar la enseñanza de la investigación en la universidad por medio de algunas piezas literarias seleccionadas por su capacidad de evocar temas de la metodología de la investigación. Los textos corresponden a autores de tres países latinoamericanos (Brasil, Argentina y Colombia) y a diferentes géneros literarios (crónica, cuento oral y manual costumbrista). Se trata de una propuesta didáctica construida a partir de una proyección, al momento sin aplicación de lo propuesto y, por tanto, sin resultados empíricos. Además de desarrollar la utilidad de tales textos, se espera promover en el docente 


\section{REVISTA DA ABRALIN}

universitario la inventiva para explorar recursos didácticos literarios no convencionales y probar sus resultados.

\section{RESUMO}

A universidade se caracteriza pela circulação e produção de certos gêneros escritos (monografias, relatórios, projetos) como uma das formas privilegiadas de avaliação dos alunos. Mesmo no caso de alunos que já passaram nos diferentes níveis de escolaridade obrigatória, as suas produções escritas revelam deficiências em termos de aspectos básicos da língua materna em geral e da língua acadêmica em particular. Esse diagnóstico afeta até mesmo alunos de cursos de ciências humanas e sociais em que a linguagem verbal é o meio usual de estudo. Essa situação é potencializada ao aprender a pesquisar, que utiliza a escrita para a socialização dos resultados. O treinamento em pesquisa geralmente ocorre entre a leitura de artigos especializados, manuais de metodologia e palestras. Sejam as fontes orais ou escritas, trata-se principalmente de textos instrutivos de marcada natureza cientificista, tendendo a imergir os alunos na objetividade e neutralidade do discurso científico. Sem pretender ignorar essa bibliografia tradicional, este artigo se propõe incentivar o ensino da pesquisa na universidade por meio de algumas obras literárias selecionadas por sua capacidade de evocar temas de metodologia de pesquisa. Os textos correspondem a autores de três países latino-americanos (Brasil, Argentina e Colômbia) e a diferentes gêneros literários (crônica, conto oral e manual costumbrista). É uma proposta didática construída a partir de uma projeção, no momento sem aplicação do que foi proposto e, portanto, sem resultados empíricos. Além de desenvolver a utilidade de tais textos, espera-se promover no professor universitário a inventividade para explorar recursos didáticos não convencionais de literatura e teste seus resultados.

\section{PALABRAS CLAVE}

Escritura. Universidad. Investigación. Literatura. Recursos didácticos.

\section{PALAVRAS-CHAVE}

Escrita. Universidade. Pesquisa. Literatura. Recursos didáticos. 


\section{REVISTA DA ABRALIN}

\section{Introducción}

La enseñanza de la investigación en la universidad discurre habitualmente entre la lectura de artículos de la especialidad, manuales de metodología y explicaciones magistrales. Sean las fuentes orales o escritas, el factor común es tratarse de textos principalmente instructivos de marcado corte cientificista, cual único camino tendiente a sumergir a los candidatos y estudiantes de doctorado en la objetividad y neutralidad del discurso científico, en las antípodas de géneros estéticos. Sin pretender ignorar esta bibliografía tradicional, el presente artículo propone abonar la enseñanza de la investigación en la universidad, en un doble sentido: proporcionando recursos didácticos no convencionales y cuestionando las fronteras entre el discurso cientificista de la academia y el estético de la literatura (GARCÍA LORENZO, 2014). En las secciones siguientes, se presentan algunos textos que, por sus características, pueden constituir otras lecturas. El orden de presentación no implica una secuencia inherente a ellos.

La propuesta se inscribe en una corriente centrada en la didáctica de la investigación y, aunque no planteada en estos términos, en la búsqueda de recursos didácticos para tal fin. Al respecto, hasta el momento la alternativa viene por el lado del género llamado "testimonios de oficios" (VALLES MARTINEZ, 2009, p. 20), que incluye todas aquellas obras que intentan develar "los entresijos, la cocina, los andamios" (WAINERMAN; DI VIRGILIO, 2010, p. 15) de la investigación. Por el contrario, las lecturas aquí elegidas no tienen ninguna intencionalidad didáctica metodológica. Sin embargo, una lectura atenta y abocada a la búsqueda de estrategias superadoras en la formación de investigadores permite identificar lecturas de potencial utilidad.

La propuesta se basa en el supuesto de que ciertos textos no científicos limítrofes (ANZALDÚA, 1987) pueden constituir recursos capaces de ayudar a los estudiantes aprender de la metáfora. Por lo tanto, el estudio crítico de cada texto se presenta en relación con el desarrollo de habilidades de investigación en la formación profesional. Otros estudios tratan de hacerlo explorando las posibilidades de las tecnologías de la información y comunicación y los medios de comunicación social (GUERIN; AITCHISON; CARTER, 2020) u otras fuentes de aprendizaje como el feedback de los supervisores y los compañeros (ZHANG; YU; YUAN, 2020). También ha sido explorado tomar los errores habituales como dispositivos de reflexión (WAINERMAN, 2009).

A partir de la importancia de la propuesta sustentada en los referentes conceptuales mencionados, a continuación se desarrolla el potencial didáctico de tres textos literarios.

\section{Lectura 1: Cómo tornar un tema costumbrista en un objeto de investigación}

Según su nombre lo indica, el Manual del asador argentino (MIRAD, 2004) consiste en un libro dedicado a transmitir sistemática y ordenadamente los conocimientos y procesos involucrados en la 


\section{REVISTA DA ABRALIN}

preparación de un asado. El libro fue elegido en tanto presenta algunas características encontradas a propósito de definir rasgos propios de la investigación científica y la escritura académica. Se presta, por tanto, a un ejercicio de argumentación por parte de los alumnos, para que respondan justificadamente si constituye o no un ejemplar de escritura académica. Previo a ello, deberán recorrerlo detenidamente. Como veremos, el análisis arrojará características que nos orientan a responder afirmativamente y otras negativamente. Aquí se resumen algunos argumentos:

El mero hecho de contenerse en un libro suele hacer pensar que sí lo es: especialmente si la encuadernación es de lomo cuadrado. En efecto, muchas personas suelen utilizar como criterio para distinguir entre un libro y una revista si el lomo es alto (cuadrado) o abrochado. Si en cambio se conociera que el libro fue comprado en una liquidación en una biblioteca de barrio, la anécdota atentaría contra la visión positiva que genera el soporte libro, pero podría obviarse y ahorrar un elemento contradictorio. Los libros son los depositarios del saber por antonomasia, aunque también sabemos que la calidad de sus contenidos es variable.

La página de legales parece confirmar esta presunción, en el sentido de que se trata de un libro registrado y que ha pasado por la entidad registradora (en este caso, la Cámara Argentina del Libro), o sea que va más allá que el trato comercial entre el autor y el editor.

La "tensión" acerca de si es escritura académica o no se produce al pasar del aspecto físico al contenido y el primer indicio de este es el título. Allí se podrá decir que el libro no constituye un ejemplar de escritura académica porque el tema no corresponde a ningún ámbito disciplinar reconocido, o al menos de los más prestigiados. Es más, se refiere a un objeto (el asado) asociado con la cultura popular argentina, lo gastronómico, que tiende a satisfacer nuestras necesidades básicas. Además, se encuentra asociado a la mera reunión de pasatiempo, o sea, bien lejano a lo culto, que uno asocia con lo académico.

Sin embargo, un examen de su interior vuelve a producir una tensión en nuestra respuesta. Su índice organizado en niveles y subniveles demuestra un gran orden. Entre los temas enumerados, presentan las siguientes características:

- uno de los temas está expresado en mayúscula, como si se tratara de un nombre propio.

- hay conceptos organizadores: categorías y sistemas.

- hay clasificaciones, que es una actividad cognitiva muy valorada.

- La expresión "el cenáculo asadiano" está formada por un término muy importante: "cenáculo", que no es un término de uso diario sino de cierta solemnidad, y por un neologismo "asadiano" que se acuña a partir del tema del libro, lo cual demuestra su importancia.

El primer capítulo empieza recurriendo al diccionario, que es una obra normativa (delimita lo que está reconocido) y de sabiduría: "Para el diccionario, Criollo es todo hijo de europeo nacido fuera 


\section{REVISTA DA ABRALIN}

de Europa, pero especialmente el americano descendiente de europeos." (p. 9). Sin embargo, se trata solo de una estrategia para impresionar, ya que, más allá de la reputación social que tenga el diccionario, no es una fuente académica de primer orden.

La sección Advertencias previas (p. 11 y 12) invoca a Borges; es decir, recurre a la literatura, y dentro de ella, a un escritor consagrado: "El Asado Criollo Tradicional, como se sabe, es un magnífico pretexto para el ritual de la "conversada amistad", como dice Borges". (p. 11). Más allá de la aparente erudición, un lector cauto pronto reconocerá que la cita textual carece de número de página, como corresponde, y tampoco está referenciado en qué obra Borges utiliza dicha expresión.

Invoca a las leyes físicas, sobre las que recuerda que son inmutables, o sea, garantía de confianza. Aquí algunos fragmentos: "la interpretación de las causas" (p. 12), que es una de las finalidades más sublimes de la investigación. "Como toda actividad doméstica ... es absolutamente empírico" (p. 12): es una forma muy elegante de conceptualizar algo que precisamente es azaroso.

Luego sigue un glosario (p. 13 y 14) como sección fundamental para evitar ambigüedades. La sección "Categorías de asadores" (p. 15 y 16) clasifica y define: "Para este último espécimen de asador está dedicado este libro". Cabe destacar que "espécimen" es un término de las ciencias naturales, que son las "ciencias madre" según la concepción positivista de la ciencia.

El capítulo "Los sistemas usados para asar" (p. 17 y 18) comienza con una exhaustiva clasificación; esto es, nada queda por fuera de ella. Esa es otra de las tareas de la ciencia: clasificar las especies. Esta desagregación en tantos niveles remite a una matriz de datos, el lenguaje de variables desarrollado por Samaja (1994) en su libro "Metodología y epistemología".

En síntesis, a la pregunta si el libro constituye una escritura académica podemos responder que sí lo es, porque es un texto riguroso y exhaustivo, aunque su tema no se corresponda con las disciplinas tradicionales.

La principal enseñanza que aporta este texto, entonces, es el de los rasgos de la escritura académica. De ellos, este texto ilustra:

- la cita de autoridad, sea apelando a intelectuales consagrados como a términos de reputación aceptada.

- la necesidad de una definición precisa de los conceptos centrales de una investigación.

- la interrelación de los conceptos, que así conforman proposiciones y, en un plano mayor, teorías.

Estos rasgos han sido desarrollados profusamente en bibliografía metodológica. Valga, a modo de ejemplos destacables, dos textos ya clásicos: el del ya mencionado filósofo argentino Juan Samaja (1994) y el de la economista y socióloga Ruth Sautu (2003) denominado, precisamente Todo es teoría. 


\section{Lectura 2: Tras la evidencia empírica y el trabajo interpretativo}

La obra "Lixo" (VERÍSSIMO, 1985), traducida al español como "Basura" o "Residuos", corresponde al género llamado crónica. Cuenta con numerosas reimpresiones, así como versiones en distintos formatos: ilustrada, en historieta, teatral ${ }^{1} \mathrm{y}$ material didáctico escolar, todo ello disponible en internet.

Esta pequeña pero encantadora obra del escritor brasileño Luis Fernando Veríssimo constituye una muestra de la capacidad humana de interpretar a partir de indicios; en este caso, de los residuos hogareños. La obra atrapa por dos razones: por la situación picaresca a través de la cual un vecino se acerca a dialogar con su vecina, y por el ingenio con el cual cada resto de basura puede reconstruir situaciones de vida. Con respecto a la primera razón: la conversación se desarrolla en el vestíbulo de un edificio, próximo al receptáculo de bolsas de residuos que es de uso común de los habitantes de un mismo piso. El lugar, y fundamentalmente el tema de conversación (la basura) no resultan ser ámbitos propios de un romanticismo. Sin embargo, en un clima de discreta cordialidad, se va entretejiendo la relación, enternecida por la caballeresca preocupación del vecino y la tierna reacción de la vecina. Con respecto a la segunda razón (la habilidad de congeniar restos de basura con situaciones de vida), se plasma en numerosas asociaciones, algunas de las cuales son las siguientes:

- La mujer ha estado deprimida porque encuentra blíster de ansiolíticos.

- Ha estado triste porque encuentra pañuelos descartables

Estas asociaciones pueden graficarse según la Tabla 1, cuyas columnas remiten a dos momentos fundamentales de la investigación: a) la recolección de información que operará de evidencia empírica y b) el trabajo interpretativo o hermenéutico en el cual se realizan las inferencias.

\begin{tabular}{|l|l|}
\hline Evidencia empírica & Inferencias \\
\hline Una tableta de ansiolíticos & Ella ha estado deprimida \\
\hline Pañuelos descartables & Ella ha estado triste \\
\hline Sobres escritos con linda letra & La madre es maestra \\
\hline
\end{tabular}

TABLA 1 - Trabajo interpretativo a partir de "Lixo" Fuente: elaborada por la autora

En la tarea de construir nuevo conocimiento en las ciencias fácticas, las conclusiones de una investigación se sustentan en la evidencia empírica obtenida a través del trabajo de campo. Este es,

\footnotetext{
${ }^{1}$ Solo brindamos a modo de ejemplo una versión teatral disponible en: https://www.youtube.com/watch?v=Rp4YBBdIsjw. El lector seguramente podrá hallar otras con toda facilidad.
} 


\section{REVISTA DA ABRALIN}

por tanto, el principal foco de enseñanza de este texto: la importancia de la evidencia empírica para arribar a conclusiones. Sin la evidencia empírica, el conocimiento permanece en un plano conjetural, hipotético, o bien en un plano metafísico como en la filosofía o en las ciencias formales (Matemática y Lógica). Ahora bien, a partir de la recolección de información, sigue una tarea fundamental cual es su interpretación. Se parte así de objetos para arribar a hechos: "Un hecho no es una cosa, ni un objeto, ni una entidad, sino más bien una situación o configuración que acontece entre entidades relacionadas de cierta manera." (KLIMOVSKY, 1998, p. 28). Este paso es el que se sintetiza en la Tabla 1 y da muestras, por tanto, del potencial didáctico del texto elegido.

\section{Lectura 3: Los riesgos de la profecía autocumplida en la investigación}

El cuento "Algo grave va a suceder en este pueblo" del escritor colombiano Gabriel García Márquez (1970) refiere a un suceso en un pequeño poblado en el que comienza a diseminarse un rumor de que algo grave sucederá allí. La sensación de tragedia irá esparciéndose entre los pobladores, quienes verán cómo la fatalidad termina ocurriendo. Lo que no pueden ver los protagonistas es que son ellos mismos quienes lo producen: perciben con estupor eventos que son cotidianos, lo que los lleva a irse del pueblo y a prenderle fuego. Al tratarse de un cuento en estilo contado contiene elementos coloquiales que proporcionan una gran empatía con los lectores. A su vez, el drama en él implícito atrapa la atención por su carácter tragicómico.

Este cuento constituye un claro ejemplo de profecía autocumplida; esto es, aquella que se cumple por sí misma. También fue llamada "efecto Pigmalión" por el sociólogo estadounidense Robert Merton (1948) a partir del escultor griego llamado Pigmalión que se enamora de su estatua Galatea. Cuando el artista conoce a Afrodita, descarga en ella todo el amor que previamente sentía por su obra, cuyo cumplimiento de la expectativa superó lo que el mismo sujeto esperaba de sí mismo (GARCÍA VARGAS, 2015, p. 41). Según esta profecía, las expectativas o creencias que los sujetos o comunidades tienen acerca de un fenómeno acaban cumpliéndose.

La profecía autocumplida se ha estudiado mucho en distintos ámbitos. En el campo de la educación se manifiesta, por ejemplo, en que los niños que mejores resultados obtienen en la escuela son aquellos cuyos docentes "profetizan" que así lo harán. A partir de dichas creencias, les dedican más atención, lo que acaba redundando en el efecto anticipado. Por el contrario, otros estudiantes, generalmente provenientes de sectores marginalizados como los inmigrantes (BALLESTÍN GONZÁLEZ, 2015), suelen fracasar puesto que no obtienen la misma dedicación que sus compañeros "privilegiados". La percepción negativa y su consecuente impacto en el rendimiento educativo no solo puede provenir de un superior (como se ha mencionado acerca de los docentes) sino también del propio estudiantado (PORCAR RARO et al., 2013) o de los padres (GARCÍA VARGAS, 2015). Afecta así a la vida subjetiva, en tanto involucra la autopercepción y los vínculos intersubjetivos. A su vez, opera 


\section{REVISTA DA ABRALIN}

reforzando prejuicios, en las antípodas de una actitud crítica. El efecto Pigmalión es aplicable a otros campos de la vida social, como es el laboral, influyendo en las expectativas de autoridades respecto de subordinados, o incluso entre pares. Otro ejemplo interesante es el del miedo a una quiebra bancaria, en un principio sin fundamento, que conduce a los clientes a retirar sus depósitos del banco llevándolo así a la bancarrota.

Por lo expresado, la profecía autocumplida puede aplicarse a la investigación, área en la que aún no ha sido explorada. La vinculación viene dada en un doble sentido: por una parte, el investigador puede autocondicionarse, y así arribar a las conclusiones con las que partía al inicio de su investigación, incurriéndose en una situación de sesgo. La investigación acaba siendo una tautología, en la que el método funciona como un mero simulacro para arribar a donde se quería llegar antes de iniciar la investigación. Por otra parte, y más propiamente en la investigación social, la profecía autocumplida se pone en juego en la forma en que el investigador interactúa con sus informantes, pudiendo condicionar también los resultados que obtiene de ellos. Dada la posibilidad de que los investigadores noveles incurran en estos errores metodológicos inintencionadamente, es importante introducir el tratamiento de estas situaciones en la enseñanza formal universitaria.

\section{Conclusiones}

El presente artículo está guiado por la necesidad de nuevas metodologías y recursos adicionales que puedan apoyar la enseñanza de la investigación en la universidad, mejorar la producción y evitar alienación (BEIGHTON, 2018). En respuesta a dicha demanda, se presentaron formas alternativas de contribuir a la alfabetización académica por medio de algunas piezas literarias seleccionadas por su capacidad de evocar temas de la metodología de la investigación. Esto es, se adoptan ciertos artefactos sociales considerados remotos y hasta incompatibles con la cultura científica como recursos de enseñanza por su ductilidad y potencial didáctico para contribuir a la educación doctoral. Específicamente, se abordan tres textos, capaces de focalizarse em determinadas facetas de la actividad investigativa: 1) la interpretación de la evidencia empírica (VERÍSSIMO, 1981); 2) las formas del discurso científico (MIRAD, 2004), y 3) el peligro de la predeterminación de los resultados (GARCÍA MÁRQUEZ, 1970). Los textos corresponden a autores de tres países latinoamericanos (Brasil, Argentina y Colombia) y a diferentes géneros literarios (crónica, cuento oral y manual costumbrista).

Las tres lecturas presentan formatos manejables para la enseñanza universitaria del quehacer de la investigación. El primero es un libro completo, pero la mera lectura de la primera parte será suficiente para encontrar ejemplos de relevancia. Las lecturas 2 y 3 , en cambio, son textos breves, y pueden ser leídos en la misma clase. "Algo grave..." presenta un grado de dramatismo que conviene plasmar en la entonación de la lectura en voz alta. "Residuos", en particular, al tratarse de un diálogo, puede ser leído entre dos compañeros. Tanto la lectura dramática como la dialogada resulta novedoso en una clase tan formalizada y por ende rompe con la dinámica habitual de la clase. 


\section{REVISTA DA ABRALIN}

Las tres propuestas están dirigidas a aquellos profesores dispuestos a revisar sus estrategias de enseñanza para superar lo que Klein (2007) denomina un abordaje meramente técnico de la escritura en la universidad. Considerando que la escritura es un componente fundamental de la investigación (DALMAGRO, 2013), una enseñanza incompleta o defectuosa de la escritura puede atentar contra el aprendizaje de la academia. Dada la complejidad que supone transmitir por escrito los resultados de una investigación científica se necesitan nuevas formas de educación superior basadas en la interdisciplinariedad: una pedagogía alternativa que contribuya a un abordaje más creativo como el aquí presentado. Sirvent y De Angelis (2011) lo refieren como "lo más desafiante y emocionante de la práctica de investigación: la creatividad científica que se desarrolla en ese "ir amasando el barro de la realidad"; es decir, que desarrollen el respeto a la realidad como nutriente de la problematización y de la generación de conocimiento científico." (p. 233)

Futuros estudios deberían relevar las respuestas de los estudiantes ante dichas propuestas y su impacto en los contenidos de aprendizaje aquí presentados. Al momento se encuentra en curso un relevamiento de dicha información a través de un cuestionario abierto que se ha implementado en una universidad pública argentina. Una vez culminada la etapa de recolección, se procederá a procesar los datos en una nueva producción académica.

Otra línea de interés que podría abrir las propuestas aquí presentadas es la búsqueda de más textos literarios que ilustren otros aspectos de la investigación.

\section{REFERENCIAS}

ANZALDÚA, G. Borderlands/La Frontera: The New Mestiza. San Francisco: Aunt Lute Books, 1987.

BALLESTÍN GONZÁLEZ, B. De "su cultura es muy fuerte" a "no se adapta a la escuela": alumnado de origen inmigrante, evaluación y efecto Pigmalión en primaria. Revista de la Asociación de Sociología de la Educación, v. 8, n. 3, p. 361-379. 2015. https://ojs.uv.es/index.php/RASE/article/view/8390/7983

BEIGHTON, C. Beyond alienation: spatial implications of teaching and learning academic writing. Teaching in Higher Education, v. 25, n. 2, p. 205-222, Dic. 2018. DOI: 10.1080/13562517.2018.1554642.

DALMAGRO, M. C. Cuando de textos científicos se trata. Córdoba, Argentina: Comunicarte, 2013.

GARCÍA LORENZO, M. M. (ed.) Estado de la cuestión sobre ciencia y literatura. Revista Signa, v. 2, n. 3 p. 15-42, 2014.

GARCÍA MÁRQUEZ, G. Algo grave va a suceder en este pueblo. Magazin Dominical, Caracas, 3 de mayo de 1970. https://www.argentina.gob.ar/sites/default/files/algo muy grave marquez.pdf.

GARCÍA VARGAS J. El efecto Pigmalión y su efecto transformador a través de las expectativas. Perspectivas docentes, n. 57, p. 40-43, 2015.

GUERIN, C.; AITCHISON, C.; CARTER, S. Digital and distributed: learning and teaching doctoral writing through social media. Teaching in Higher Education, v.5, n 2, p. 238-254, 2019. DOI:10.1080/13562517.2018.1557138 


\section{REVISTA DA ABRALIN}

KLEIN, I. (coord.). El taller del escritor universitario. Buenos Aires: Prometeo Libros, 2007.

KLIMOVSKY, G. La inexplicable sociedad. Buenos Aires: AZ editores, 1998.

MERTON, R. K. The Self Fulfilling Prophecy. Antioch Review, v. 8, n. 2, p. 193-210, 1948. doi:10.2307/4609267

MIRAD, R. Manual del asador argentino. Alicante, España: Club Universitario, 2004.

PORCAR RARO, E.; GIMENO NÁCHER. M. E.; BALAGUER RODRÍGUEZ, P.; ALEDÓN PITARCH, B. El efecto Pigmalión, autoestima y rendimiento escolar. Fòrum de recerca, n. 18. p. 417-422. 2013.

http://dx.doi.org/10.6035/ForumRecerca.2013.27

SAMAJA, J. Epistemología y metodología. Elementos para una teoría de la investigación científica. Buenos Aires: Eudeba, 1994.

SAUTU, R. (2003). Todo es teoría. Objetivos y métodos de investigación. Buenos Aires: Lumiere.

SIRVENT, M. T. y DE ANGELIS S. Pedagogía de formación en investigación: la transformación de las conceptualizaciones acerca de la investigación científica. Cuadernos de Educación, año IX, n. 9, p. 231-244, 2011.

VALLES MARTINEZ, M. Hacerse investigador social: testimonios de oficio y artesanía intelectual del sociólogo. Política y Sociedad, v. 46, n. 3, p. 13-36, 2009. Disponible en: https://revistas.ucm.es/index.php/POSO/article/view/POSO0909230013A/21746.

VERÍSSIMO L. F. Lixo. En O Analista de Bagé. Porto Alegre: L\&PM, 1981.

WAINERMAN, C. Introducción: acerca de la formación de investigadores en ciencias sociales. In: WAINERMAN, C.; SAUTU, R. (comp.). La Trastienda de la Investigación. Buenos Aires: Lumiere, 3a ed., 2001, p. 3-16. Disponible en: https://www.fapyd.unr.edu.ar/wp-content/uploads/2015/09/Wainerman-Sautu-indice-cap-1.pdf.

WAINERMAN, C.; DI VIRGILIO, M. M. El quehacer de la investigación en educación. Buenos Aires: Manantial, 2010.

ZHANG, Y.; YU, S.; YUAN, K. Understanding Master's students' peer feedback practices from the academic discourse community perspective: A rethinking of postgraduate pedagogies, Teaching in Higher Education, v. 25, n. 2, p. 126-140, 2020. DOI: 10.1080/13562517.2018.1543261 